\title{
Photometric study of eclipsing dwarf nova EX Dra
}

\author{
Irina Voloshina*t \\ Moscow State university, Sternberg Astronomical Institute, Universitetsky prospect 13, Moscow \\ 119234, Russia \\ E-mail: voloshina.ira@gmail.com
}

\section{Tatiana Khruzina}

Moscow State university, Sternberg Astronomical Institute, Universitetsky prospect 13, Moscow 119234, Russia

E-mail: kts@gmail.com

\section{Vladimir Metlov}

Moscow State university, Sternberg Astronomical Institute, Universitetsky prospect 13, Moscow 119234, Russia

E-mail: vgmetlov@gmail.com

We have analysed long-term photometric observations of the dwarf nova EX Dra, a system with a long orbital period, in quiescence, the pre-outburst state, and the active state. The aim of our study was to test the hypothesis of Baptista [2012, MmSAI, 83, 530] that the outbursts in this system are due to an increasing rate of mass outflow from the secondary rather than the thermal instability associated with changing viscosity in the disk

The Golden Age of Cataclysmic Variables and Related Objects V (GOLDEN2019)

2-7 September 2019

Palermo, Italy

\footnotetext{
* Speaker.

${ }^{\dagger}$ This study has been supported via RFBR grant No 17-52-53200
} 


\section{The brief history of EX Dra system}

Cataclysmic variable EX Dra (HS1804+6753) with coordinates $\alpha=18^{h} 04^{m} 14^{s} .11$ and $\delta=$ $+67^{\circ} 54^{\prime} 12.2^{\prime \prime}$ was detected in the Hamburger Quasar Survey [Bade et al., 1989]. It was shown by Barwig et al. [1993] to be an eclipsing dwarf nova with deep eclipses $\sim 1.5^{m}$. Outbursts occur every 10-30 days with duration about 10 days and amplitude up to 2-3 $3^{m}$. EX Dra brightness reaches $\sim 13.5^{m}$ at the outburst and is about $\sim 15^{m}$ during quiet state. It is a system with a long orbital period (about 5 hours).

Masses of the components were determined from spectroscopic observations by Baptista et al. [2000]. They are: $M_{1}=(0.75 \pm 0.15) M_{\odot}, M_{2}=(0.54 \pm 0.10) M_{\odot}, q=1.39 \pm 0.12, i \sim 85^{\circ} \pm 2$. Spectrum of the secondary component is estimated as M1V - M2V, its absolute magnitude as $8.8<M_{V}(2)<9.7$, the distance $\sim 290 \pm 80 \mathrm{pc}$ (all parameters were taken from Baptista et al. [2000])

\section{Observations}

Observations of the system were obtained in 2014-2016 with the help of CCD photometers mounted on the two telescopes, 50 and $60-\mathrm{cm}$, in Crimea during both quiet and the outburst state. One of them (used at the 50-cm telescope) has an Apogee Alta U8300 CCD detector. The other one used at the 60-cm telescope has an Apogee 47 detector. Observations were done in the red $\left(R_{c}, \lambda=\right.$ $6700 \mathrm{~A}$ ) because the sensitivity of both light detectors was the highest in this particular range. The length of the observational sets mostly exceeded $5 \mathrm{~h}$ with uncertainty of a single measurement $\sigma$ about $0.02-0.06^{m}$. The star from the vicinity of EX Dra with magnitudes $V=12^{m} .038, R_{c}=$ $11^{m} .832$ and coordinates $\alpha=18^{h} 03^{m} 37^{s} .55, \delta=+67^{\circ} 57^{\prime} 01.7^{\prime \prime}$ was used as a local standard. The fluxes of EX Dra relative this star were determined. The constance of the comparison star was verified using a sample of the so-called "check stars" every night of our observations. 24 observational sets at all were obtained with the 50-cm telescope in 2014-2015 and 7 sets - with the 60-cm telescope in 2015-2016. 2 series of simultaneous observations were provided on both telescopes to find any systematic difference between the measured fluxes. Our telescopes and photometers are shown in Fig.1. It was found that the fluxes of EX Dra obtained with the 50$\mathrm{cm}$ and $60-\mathrm{cm}$ telescopes are related according to the equation $R_{c}(50)=R_{c}(60)+0^{m} .05$. For the further analysis, all fluxes were reduced to the $50-\mathrm{cm}$ instrumental system. The time distribution of all our observations of dwarf nova EX Dra in $R_{c}$ band is shown in Figure 2.

\section{Light curves of EX Dra}

The light curves were constructed using our observations for the different states of activity of EX Dra. One can see some of them in Figure 3 and 4 separately for quiescence and outburst. A consideration of these light curves shows that as a whole, the shapes of the light curves of EX Dra obtained in quiescence and during outburst are typical of a dwarf nova. 

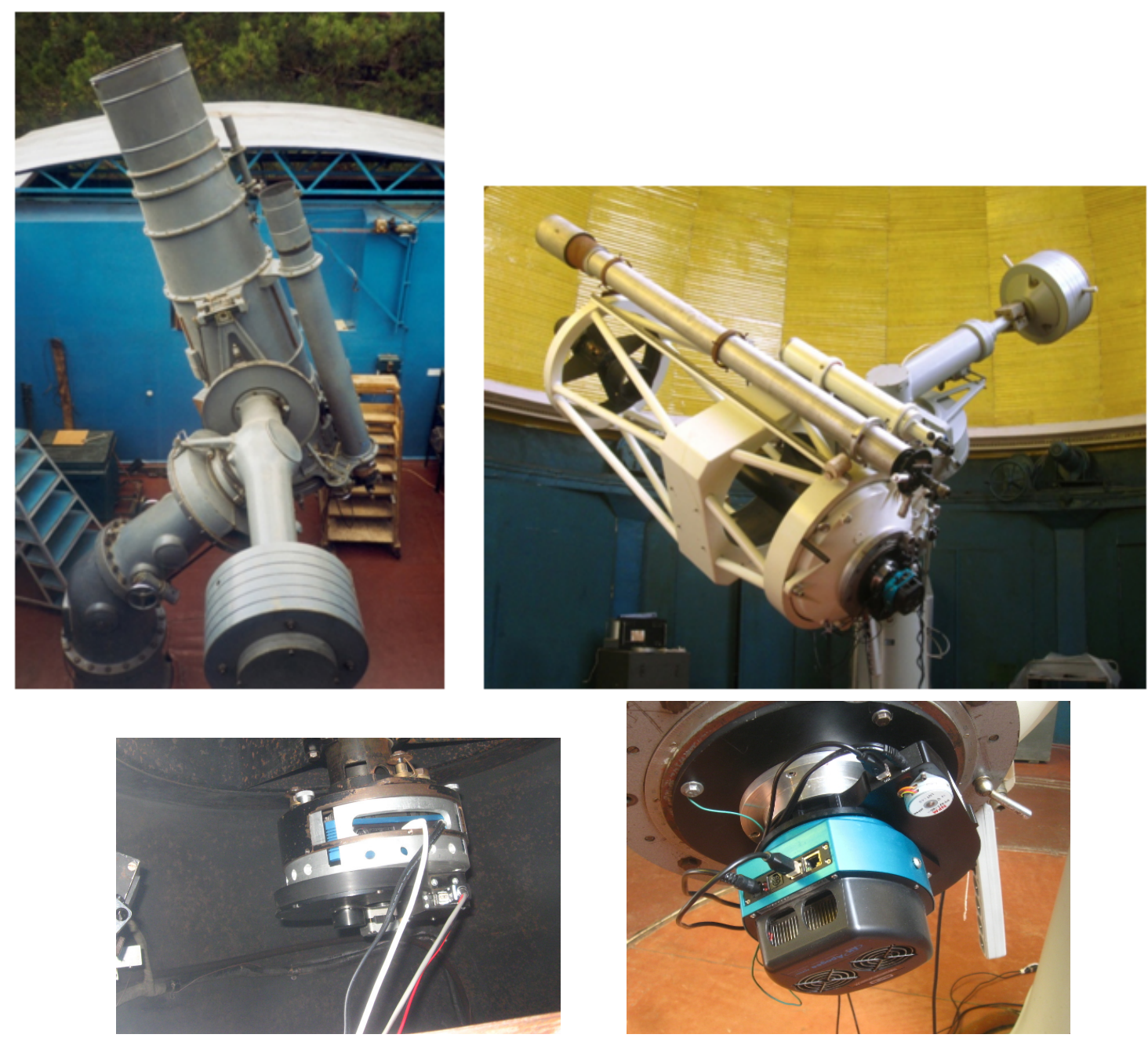

Figure 1: Telescopes and photometers which were used for providing our observations

\section{Orbital period determination}

We searched for the orbital period of EX Dra using all the available data. This procedure was performed using the Lafler-Kinman method. The period derived from our $R_{c}$ observations is $P_{o r b}=0^{d} .2099366(6)$. The resulting period is consistent within the errors with the period from the article Pilarcik et al. [2012]. Figure 5 displays the power spectrum derived for the $R_{c}$ observations.

\section{Models used for fitting}

In our study all parameters of the system (its accretion disk,gaseous stream, hot line, hot spot, and other components) are derived in a "combined model", worked out for cataclysmic variables. This model takes into account visual manifestations of the interaction between the gas flow and the accretion disk in a close binary system in the form of a "hot line" and a "hot spot". The detailed description of this model one can find in Khruzina [2011]. The binary consists of a red dwarf that fills its Roche lobe and a compact spherical star (a white dwarf) surrounded with a thick 
ellipsoidal accretion disk of a complex shape. The disk thickness is not large near the compact star but increases according to a parabolic law towards its outer edge. The oblique collision of the gaseous flow with matter of the cool, rotating disk, whose outer edge has a temperature $<10000$ $\mathrm{K}$, creates an extended region of enhanced energy release. In the combined model, this region is represented with a hot line that coincides with the optically opaque part of the flow. It is located outside the disk, together with a hot spot at the outer surface of the disk, on the leeward side of the flow. This model is able to reproduce the shapes of both classical and atypical light curves of CV-s both in the active state and in quiescence.

To estimate the degree of agreement between the theoretical and observed light curves of the close binary in the model used, we calculated the $\chi^{2}$ residuals in the form

$$
\chi^{2}=\sum_{j=1}^{J} \frac{\left(m_{j}^{\text {theor }}-m_{j}^{\text {obs }}\right)^{2}}{\sigma_{j}^{2}},
$$

where $m_{j}^{\text {theor }}$ and $m_{j}^{o b s}$ are the magnitudes of the object at orbital phase $j$ derived theoretically and from the observations, $\sigma_{j}$ is the dispersion of the observations for point $j$, and $J$ is the number of normal points in the mean light curve. A consideration of a few of the light curves (for example, JD 6834) shows that the secondary minimum, though symmetric, is located in the orbital phase $\varphi \sim 0.6$ (this is especially obvious for the light curve for JD 7167); the out-of-eclipse part of this light curve for JD 6834 even shows two local minima (see Fig. 6, left panel). Such displacements cannot be described in the standard combined model, since the hot spot is screened by the disk edge at these phases, and the gaseous stream is not visible at phases $\varphi \sim 0.5$ for $i>83^{\circ}$. The observed shift of the secondary minimum, and also the dips of varying depth in the light curves, can be reproduced if the contribution of the light from the secondary of the total flux is asymmetric. This effect can be created by the presence of one or two cool spots on the stellar surface.

\section{Determination of EX Dra parameters}

All parameters of the EX Dra system (its accretion disk, gaseous stream, hot line, hot spot, and other components) were derived in combined model for cataclysmic variables described above. Some of them are collected in Table I as an example. The contribution of components to the total flux of the EX Dra system for both states is shown at the bottom of this Table. They are very effectively different from each other, clearly demonstrating that during an outburst, $2 / 3$ of the total flux are caused by the radiation of the accretion disk due to an increase in size, a flatter temperature distribution along its radius, and a higher temperature in its inner layers.

\section{Summary of results}

We analysed long-term CCD observations of dwarf nova EX Dra in quiescence, the preoutburst state, and the active state obtained at the Crimean Astronomical Station of the Sternberg Astronomical institute in 2014-2016. Our analysis of all these observations permits us to conclude the following. 
Table 1: Variations of accretion disk and hot line parameters of EX Dra in $R c$ band

\begin{tabular}{|l|c|c|}
\hline Parameter & Inactive state & Active state \\
\hline$R_{d}, a_{0}$ & $0.16-0.32$ & $0.31-0.36$ \\
$e$ & $0.003-0.3$ & $0.003-0.3$ \\
$0.5 \beta_{d},{ }^{\circ}$ & $0.5-1.7$ & $0.6-1.6$ \\
$T_{i n}, \mathrm{~K}$ & $19100-25700$ & $24200-33400$ \\
$\gamma$ & $0.43-0.58$ & $0.24-0.42$ \\
$T_{w w}, \mathrm{~K}$ & $29000-75600$ & $33600-86500$ \\
$T_{l w}, \mathrm{~K}$ & $31800-66500$ & $30600-78900$ \\
\hline Components' contribution to the total flux from the system $F_{\text {full }}$ \\
\hline Star & $0.40-0.56$ & $0.18-0.28$ \\
WD & $0.07-0.11$ & $0.02-0.05$ \\
Disk with HS & $0.22-0.52$ & $0.66-0.77$ \\
HL & $0.01-0.07$ & $0.02-0.05$ \\
\hline
\end{tabular}

Comments. $T_{i n}$ — temperature of inner regions of the disk; $T_{w w}$ — temperature of windward side of gaseous stream; $T_{l w}-$ temperature of leeward side of gaseous stream; $\beta_{d}$ - thickness of the outer edge of accretion disk. The bottom of the table shows the contributions of components to the total flux from the system.

- The orbital period of EX Dra was derived from our photometric observations which confirmed the value found earlier $-P_{o r b}=0^{d} .2099366(6)$.

- A combined model taking into account the presence of a hot spot on the lateral surface of the accretion disk and the contribution of light from the gaseous stream near the outer edge of the disk can successfully be applied to determine the parameters of EX Dra in different states of the system's activity for most of the studied light curves.

- Six observed light curves could not be satisfactorily fit with theoretical ones in frame of this model. Accordingly, we added one or two dark spots on the surface of the red dwarf secondary to the combined model. Taking in to account the existence of these spots, we were able to qualitatively reproduce the existence of secondary minima at phases differing from $\varphi \sim 0.5$.

- The analysis of our observations of the cataclysmic variable EX Dra testifies that this system is a dwarf nova with MTIM-driven outbursts (MTIM - Mass Transfer Instability Model). This is due to the high viscosity of the matter in the accretion disk, small changes in the viscosity in the transition from quiescence to the active state, and the contraction of the accretion disk and increase of the radiation from the hot spot and hot line prior to outburst.

- So the main conclusion of our study - the outbursts in the EX Dra system are related to instability of the matter outflow from the secondary (i.e. MTIM). 


\section{Acknowledgments}

This work was supported by the Russian Foundation for Basic Research via grant No. 17-5253200 and by a grant from the Development Program of the Lomonosov Moscow State University for the Leading Scientific School "Physics of Stars, Relativistic Objects, and Galaxies".

The authors are grateful to V. Sementsov, $\mathrm{PhD}$ for a fruitful discussion.

\section{References}

[1] Bade, N., Hagen, H.-J., and Reimers, D., in Proceedings of the 23rd ESLAB Symposium, Ed. by J. Hunt and B. Battrick, ESA SP-296 (ESA, Noordwijk, 1989), p. 883.

[2] Baptista, R., Mem. Soc. Astron. Ital. 83, 530 (2012).

[3] Baptista, R., \& Catalán, M. S. Astrophys. J. Lett. 539, L55 (2000).

[4] Barwig, H., Fiedler, H., Reimers, D., and Bade, N., in IAU Symposium 165: Compact Stars in Binary Systems, Ed. by H. vanWoerden (Kluwer, Dordrecht, 1993), p. 89.

[5] Khruzina, T. S., Astron. Rep. 55, 425 (2011).

[6] Khruzina, T. S. and Cherepashchuk, A. M., Sov. Astron. 39, 178 (1985).

[7] Pilarčík, L., Wolf M., Dubovský, P. A., Hornoch, K., Kotková, L., Astron. Astrophys. 539, A153 (2012).

\section{DISCUSSION}

DAVID BUCKLEY: In other systems that have MTIM outbursts they show enhanced S-wave in the trailed spectra due to the increased hotspot emission. Do the spectra of EX Dra shows enhanced S wave in outburst?

IRINA VOLOSHINA: I find only one old paper of Fiedler [H. Fielder, H. Barwig, and K.H. Mantel, Astron. and Astrophys., 327, 173 (1997)].In this paper one can probably see S-wave but in absorption lines (in Fig.4). They also write that plan to continue their study of object spectra later but no further results were published. 

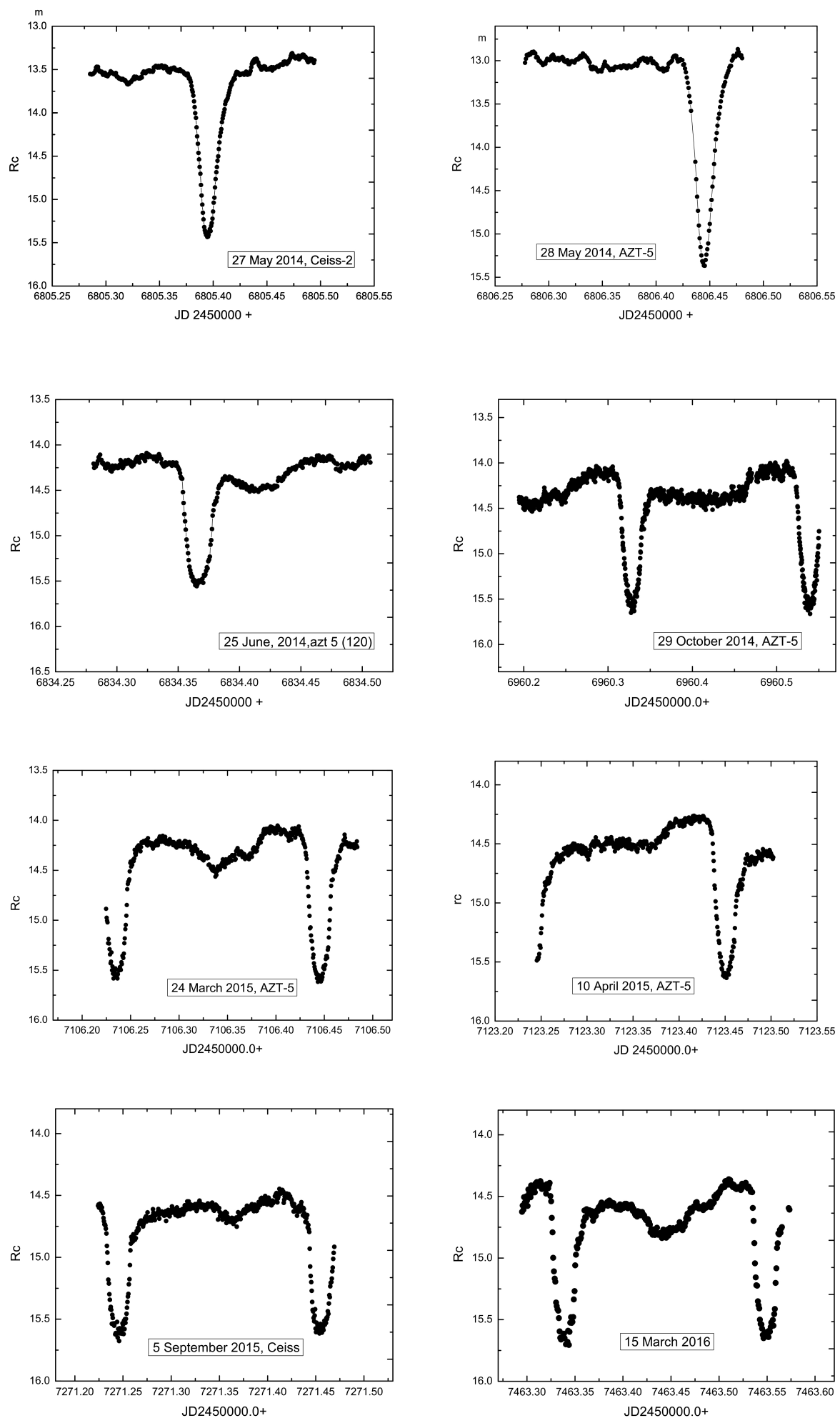

Figure 2: Some of EX Dra light curves obtained at the different observational seasons, in quiescence 

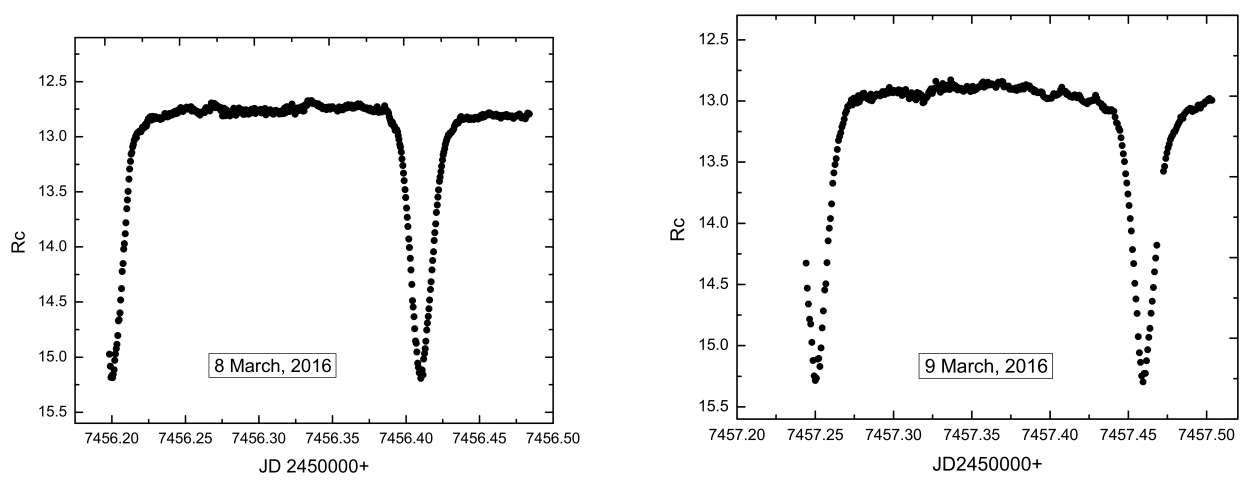

Figure 3: EX Dra observations at outburst

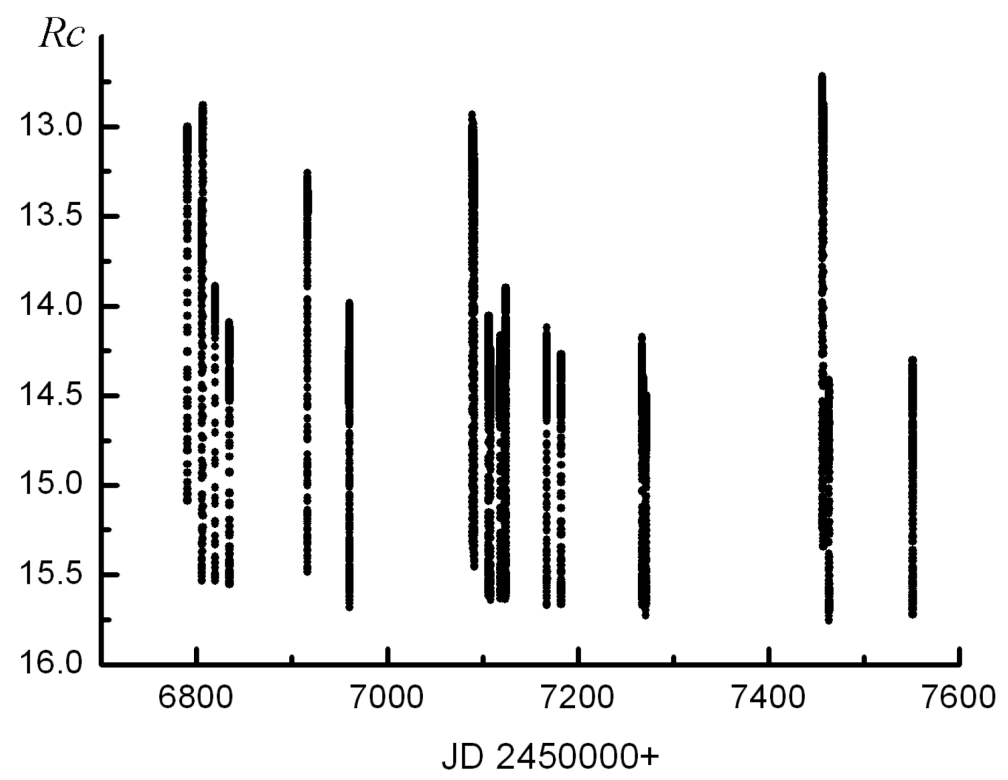

Figure 4: The overall light curve of EX Dra. Plot shows changes of system brightness during the period of our observations in the $R_{c}$ filter. 


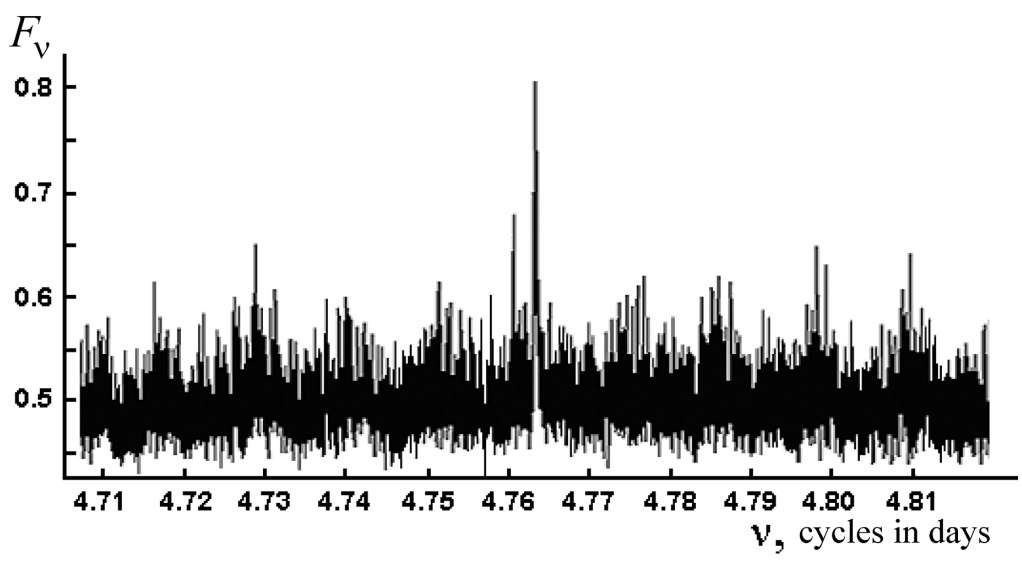

Figure 5: The Lafler-Kinman power spectra constructed using the $R_{c}$ observations. The highest peak corresponded to the orbital period $P_{\text {orb }}=0.2099366(5)^{d}$
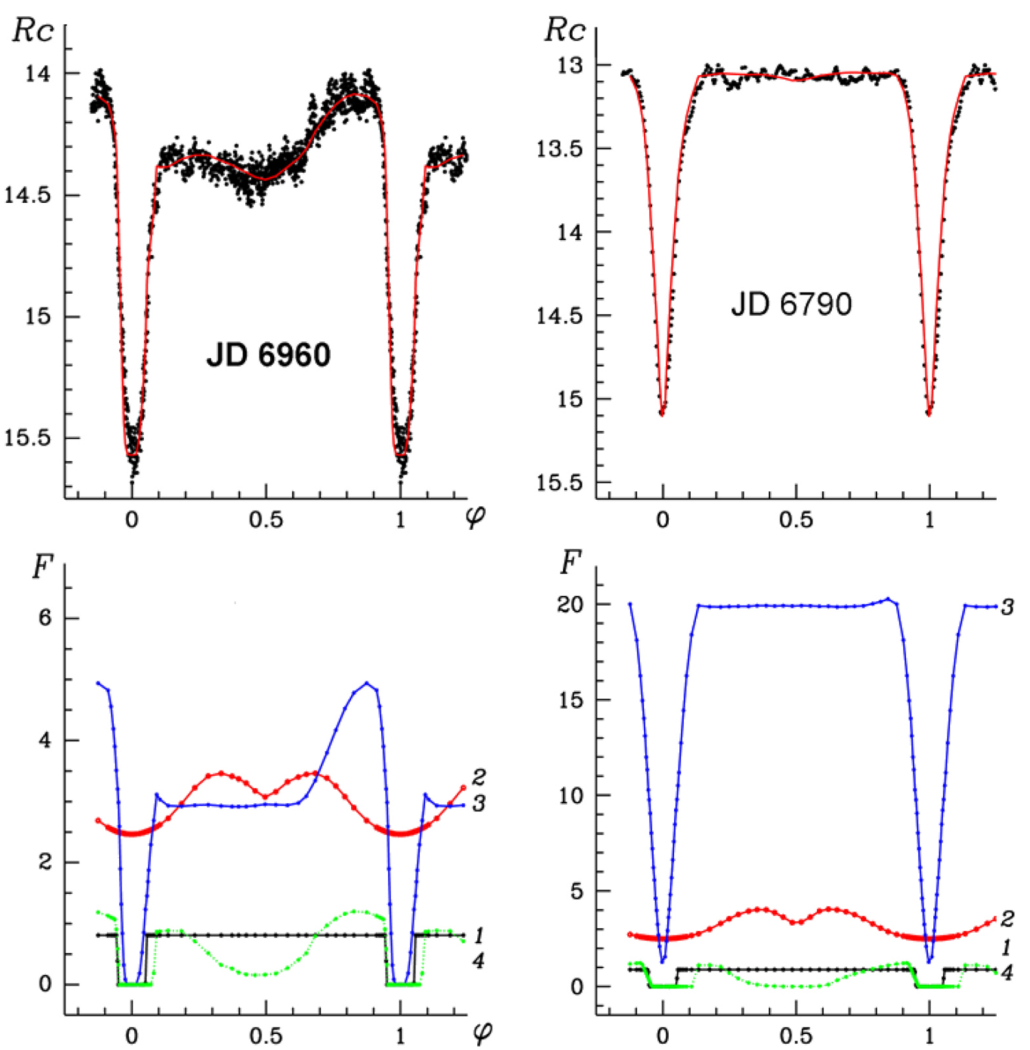

Figure 6: Results of our analysis for the typical light curves of EX Dra: left panels show light curves during quiescence, right panels - during outburst. Observations are shown by points, theoretical curves - red line. Contribution of various components to the total flux of the system are shown on the two low panels for accordingly: 1 - white dwarf; 2 - red dwarf; 3 - disk with hot spot; 4 - hot line. 

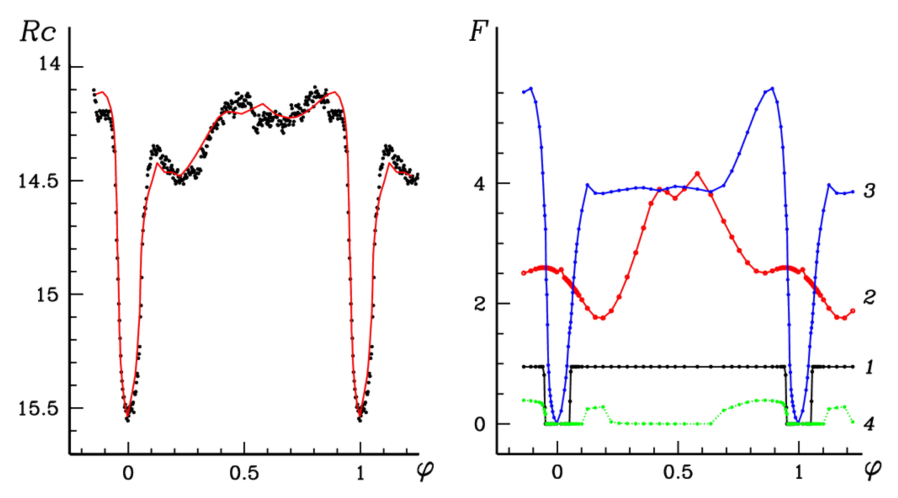

JD 6834

Figure 7: Results of provided analysis for one of the abnormal observational light curves as an example. Left panel: observations - points, the theoretical curve - red line. Contribution of different components to the total flux of the system are shown on the central panel for accordingly: 1 - white dwarf; 2 - red dwarf; 3 - disk with hot spot; 4 - hot line. A schematic representation of such a system is shown on the right. 